\title{
A rare complication of ERCP: duodenal perforation due to biliary stent migration
}

\section{(ㄷ)(1) $\odot$}

\author{
Authors \\ Lehman', Evan L. Fogel ${ }^{1}$ \\ Institutions \\ 1 Division of Gastroenterology and Hepatology, Indiana \\ University School of Medicine, Indianapolis, IN, USA \\ 2 Indiana University School of Medicine, Indianapolis, IN, \\ USA
}

Mark A. Gromski ${ }^{1}$, Benjamin L. Bick ${ }^{1}$, David Vega ${ }^{2}$, Jeffrey J. Easler ${ }^{1}$, James L. Watkins ${ }^{1}$, Stuart Sherman ${ }^{1}$, Glen A.

submitted 18.12 .2019

accepted after revision 11.5 .2020

\section{Bibliography \\ Endoscopy International Open 2020; 08: E1530-E1536 \\ DOI 10.1055/a-1231-4758 \\ ISSN 2364-3722 \\ (c) 2020. The Author(s).}

This is an open access article published by Thieme under the terms of the Creative Commons Attribution-NonDerivative-NonCommercial License, permitting copying and reproduction so long as the original work is given appropriate credit. Contents may not be used for commecial purposes, or adapted, remixed, transformed or built upon. (https://creativecommons.org/licenses/by-nc-nd/4.0/)

\section{Corresponding author}

Evan L. Fogel, MD, MSc, Indiana University School of Medicine, University Hospital, Suite 1602, 550 University Blvd, Indianapolis, IN 46202-5250, United States

Fax: +1-317-968-1265

efogel@iu.edu

\section{ABSTRACT}

Background and study aims Perforation of the duodenal wall opposing the major papilla due to a migrated pancreatobiliary stent rarely has been described in the literature as a complication of endoscopic retrograde cholangiopancreatography (ERCP). Factors associated with perforation from migrated stents from ERCP are unknown.

Patients and methods This was a retrospective, observational study. Patients were identified from January 1, 1994 to May 31, 2019 in a prospectively maintained ERCP database.

Results Eleven cases of duodenal perforation from migrated pancreatobiliary stents placed at ERCP were identified during the study period. All cases involved biliary stents, placed for biliary stricture management. The perforating stent was plastic in 10 cases $(91 \%)$. This complication occurred in one in 2,293 ERCP procedures in which a pancreatobiliary stent was placed.

Conclusion This complication is more common with biliary stents compared to pancreatic stents. This may be related to the angle of exit of biliary stents being more perpendicular to the opposing duodenal wall and the near exclusive use of external pigtail plastic stents in the pancreatic duct. All perforating plastic stents were $\geq 9 \mathrm{~cm}$ in length. Longer stents may provide leverage for perforation with a migration event.

\section{Introduction}

Placement of pancreatobiliary stents during endoscopic retrograde cholangiopancreatography (ERCP) is a common practice. Stents can be used for management of various entities, including malignant biliary strictures, large obstructing bile duct or pancreatic duct stone burden, benign biliary or pancreatic duct strictures, bile leak, papillary stenosis, pseudocyst drainage, and prevention of post-ERCP pancreatitis. Migration of a biliary stent is a known potential complication of ERCP, with distal migration occurring in $4 \%$ to $6 \%$ of cases $[1,2]$.

Perforation of the duodenal wall opposing the major papilla due to a migrated pancreatobiliary stent has been described previously in the literature as a complication of ERCP-nearly universally in case reports [3-15]. From all accounts, it is a rare complication, yet the adverse outcome from a duodenal perforation can be devastating. Factors associated with perforation from migrated stents are unknown. Furthermore, the incidence rate of this complication is also not currently appreciated.

The primary aims of this study were to describe cases of duodenal perforation from migrated pancreatobiliary stents and to identify potential risk factors that may lead to perforation. 


\section{Patients and methods}

This study was approved by the hospital Institutional Review Board (IRB). Patients were identified retrospectively from our prospectively maintained institutional ERCP database. Patients were identified from January 1, 1994 to May 31, 2019. The database was queried for patients with a finding of perforation at time of endoscopy. Furthermore, endoscopists within the advanced endoscopy group at Indiana University Hospital who perform ERCP were queried to capture any internally collected cases of migrated pancreatobiliary stents causing duodenal perforation. A retrospective review of clinical information from the electronic medical record (Cerner, North Kansas City, Missouri, United States) was performed for each identified patient. Patients were included if there was documented evidence of a pancreatobiliary stent causing duodenal perforation. Demographics and clinical data for included patients were collected from the electronic medical record and the electronic endoscopy reporting system (ProVation, Minneapolis, Minnesota, United States). Fluoroscopic and endoscopic images were reviewed from the ERCPs that met the inclusion criteria.

The type, size, and location of placement of endoscopic pancreatobiliary stents were at the discretion of the individual endoscopist. At our institution, the endoscopist will frequently measure the length of the area to be stented with an endoscopic tool that can be visualized fluoroscopically, such as an ERCP catheter, stent guide catheter or a stone extraction balloon. Some endoscopists manually groom the plastic biliary stents with gentle heat to have the shape and bend of the stent conform more accurately with the shape of the proximal bile duct.

\section{Results}

During the study period, 25,224 ERCP procedures with endoscopic stent placement were performed at our institution. Eleven cases of duodenal perforation from migrated pancreatobiliary stents placed at ERCP were identified during the study period. Thus, the rate of this complication occurring is one in 2,293 $(0.04 \%)$ ERCP procedures in which a pancreatobiliary stent was placed.

Eight of the 11 patients had some symptom or finding that could be attributable to a viscus perforation (e.g., abdominal pain, sepsis or leukocytosis). Three patients had no abdominal symptoms and had ERCP for routine stent exchange. All 11 cases involved transpapillary biliary stents. There were no cases identified of pancreatic stent migration causing duodenal perforation. Demographics, indication for original ERCP, findings at ERCP, type of stent, location of perforation, treatment strategy for perforation, and potential risk factors for perforation are detailed in $>$ Table 1 . The mean age of patients in this series was 67.1 years (SD 17.4). Findings at ERCP included a biliary stricture in all 11 cases. Four patients had a stricture located at the common hepatic duct, two had a stricture of the common bile duct (CBD), two had multifocal intrahepatic and extrahepatic biliary strictures, one had a stricture of the left main intrahepatic duct, and two had Bismuth IV hilar strictures. The perfor- ating stent was a plastic biliary stent in 10 cases (91\%), with the proximal end of the stent terminating in an intrahepatic duct in nine cases and the common hepatic duct in one case at the time of stent placement. Plastic biliary stents used had a bend factory-groomed in the middle of the stent (i.e., Cotton-Leung biliary stent [Cook Medical, Bloomington, Indiana, United States]). Of the nine intrahepatic stents causing perforation, eight ( $89 \%$ ) had no documentation of being manually groomed to conform to the trajectory of the intrahepatic ducts. However, in this retrospective review, it remains possible that stent grooming may have taken place but was simply not mentioned by the endoscopist in the ERCP procedure report. Of the seven patients who had bilateral intrahepatic biliary stents placed, the culprit stent was left-sided in six cases (86\%). The stent perforation was discovered at a mean of 37.4 days (SD 37.0) after stent placement. All but two cases had multiple biliary stents placed at the time of implicated ERCP. > Fig. 1 and > Fig. 2 show the endoscopic identification and treatment of duodenal perforations from a migrated stent. > Fig. 3 is an intraoperative image illustrating transmural migration of a plastic biliary stent.

- Table 2 illustrates the clinical follow-up for these patients. Three patients are known to have died, one 19 days after the ERCP (index procedure resulting in perforation) from sepsis, one 155 days after ERCP after having been placed in hospice, and another 31 days after ERCP after having been placed in hospice. Another patient was discharged home from the hospital 11 days after ERCP with comfort measures only and was subsequently lost to follow-up. Of these four patients, three were elderly (> 70 years old) and all had an advanced primary pancreatobiliary malignancy. Of the three patients with benign disease in this series (post-cholecystectomy biliary stricture, necrotizing pancreatitis and primary sclerosing cholangitis), they remain alive after follow-up of 1610,1193 , and 673 days, respectively.

\section{Discussion}

This case series describes a rare but serious complication of ERCP: biliary stent migration resulting in perforation of the opposing duodenal wall. Although it is difficult to attribute a single complication to subsequent events in patients with significant comorbid disease, such as end-stage pancreatobiliary malignancy, it is plausible that this complication at least precipitated an earlier than expected death in three patients in this series.

In our series, this complication was more common with biliary stents compared to pancreatic stents, with no cases noted of a migrated pancreatic duct stent causing duodenal wall perforation. This may be related to the axis of the luminal end of biliary stents being more perpendicular to the opposing duodenal wall compared to pancreatic duct stents. Furthermore, at our center, we exclusively place stents with external pigtails into the pancreatic duct. Straight (non-pigtail) pancreatic stents are not used at our center. Pigtail stents may be less traumatic than straight stents as they have no leading "point," should the stent migrate. All but one of the migrated biliary 
- Table 1 Clinical and procedural characteristics of patients with duodenal perforation attributable to biliary stent migration.

\begin{tabular}{|c|c|c|c|c|c|c|c|c|}
\hline $\begin{array}{l}\text { Patient, } \\
\text { age } \\
\text { (Years), } \\
\text { Gender }\end{array}$ & $\begin{array}{l}\text { Indica- } \\
\text { tion for } \\
\text { ERCP }\end{array}$ & $\begin{array}{l}\text { Pancrea- } \\
\text { tobiliary } \\
\text { Diagnosis }\end{array}$ & $\begin{array}{l}\text { ERCP } \\
\text { Findings }\end{array}$ & $\begin{array}{l}\text { Pancreatobiliary } \\
\text { Stents Used } \\
\text { (Stent Diameter, } \\
\text { Stent Length) }\end{array}$ & $\begin{array}{l}\text { Time to } \\
\text { Perfora- } \\
\text { tion Iden- } \\
\text { tification } \\
\text { (Days } \\
\text { Post- } \\
\text { ERCP) }\end{array}$ & Location & $\begin{array}{l}\text { Treatment } \\
\text { of Perfora- } \\
\text { tion }\end{array}$ & $\begin{array}{l}\text { Potential } \\
\text { Risk Factors }\end{array}$ \\
\hline $\begin{array}{l}\# 1,58, \\
\text { female }\end{array}$ & $\begin{array}{l}\text { Abdomi- } \\
\text { nal pain, } \\
\text { biliary } \\
\text { stent ex- } \\
\text { change }\end{array}$ & $\begin{array}{l}\text { Post-cho- } \\
\text { lecystect- } \\
\text { omy biliary } \\
\text { stricture }\end{array}$ & $\begin{array}{l}\text { Common he- } \\
\text { patic duct } \\
\text { stricture }\end{array}$ & $\begin{array}{l}10 \mathrm{Fr}, 9 \mathrm{~cm} \text { plastic } \\
\text { biliary stent in } \\
\text { right hepatic duct } \\
\mathbf{1 0} \mathrm{Fr}, \mathbf{1 1} \mathbf{c m} \text { plas- } \\
\text { tic biliary stent in } \\
\text { left hepatic duct }{ }^{1}\end{array}$ & 19 & $\begin{array}{l}\text { Retroperito- } \\
\text { neal }\end{array}$ & $\begin{array}{l}\text { Stent re- } \\
\text { moval } \\
\text { Endoscopic } \\
\text { clip (3) clo- } \\
\text { sure } \\
\text { Percuta- } \\
\text { neous drain } \\
\text { for retroper- } \\
\text { itoneal ab- } \\
\text { scess }\end{array}$ & $\begin{array}{l}\text { Chronic rheuma- } \\
\text { toid arthritis on } \\
\text { immunosuppres- } \\
\text { sive therapy } \\
\text { (methotrexate, } \\
\text { infliximab, pre- } \\
\text { dnisone); peri- } \\
\text { ampullary diver- } \\
\text { ticulum }\end{array}$ \\
\hline $\begin{array}{l}\# 2,40, \\
\text { male }\end{array}$ & $\begin{array}{l}\text { Jaundice, } \\
\text { CT abnor- } \\
\text { mality }\end{array}$ & $\begin{array}{l}\text { Metastatic } \\
\text { abdominal } \\
\text { desmo- } \\
\text { plastic } \\
\text { small } \\
\text { round cell } \\
\text { tumor with } \\
\text { biliary ob- } \\
\text { struction }\end{array}$ & $\begin{array}{l}\text { Common he- } \\
\text { patic duct } \\
\text { stricture }\end{array}$ & $\begin{array}{l}10 \mathrm{Fr}, 15 \mathrm{~cm} \text { plas- } \\
\text { tic biliary stent in } \\
\text { left hepatic duct, } \\
\text { groomed }{ }^{1} \\
10 \mathrm{Fr}, 12 \mathrm{~cm} \text { plastic } \\
\text { biliary stent in } \\
\text { right hepatic duct }\end{array}$ & 2 & Peritoneal & $\begin{array}{l}\text { Stent re- } \\
\text { moval } \\
\text { Nasoduode- } \\
\text { nal suction } \\
\text { and bowel } \\
\text { rest } \\
\text { Percuta- } \\
\text { neous drain } \\
\text { for abdomi- } \\
\text { nal abscess }\end{array}$ & $\begin{array}{l}\text { History of radia- } \\
\text { tion and ongoing } \\
\text { chemotherapy } \\
\text { with pazopanib; } \\
\text { cancer }\end{array}$ \\
\hline $\begin{array}{l}\# 3,88 \text {, } \\
\text { female }\end{array}$ & Jaundice & $\begin{array}{l}\text { Pancreatic } \\
\text { adenocar- } \\
\text { cinoma } \\
\text { with biliary } \\
\text { obstruc- } \\
\text { tion }\end{array}$ & $\begin{array}{l}\text { Distal com- } \\
\text { mon bile duct } \\
\text { stricture }\end{array}$ & $\begin{array}{l}10 \mathrm{~mm}, 6 \mathrm{~cm} \text { un- } \\
\text { covered metal bili- } \\
\text { ary stent in com- } \\
\text { mon bile duct } \\
10 \mathrm{~mm}, 4 \mathrm{~cm} \text { un- } \\
\text { covered metal } \\
\text { biliary stent } \\
\text { placed } 11 \text { months } \\
\text { later, within in- } \\
\text { terstices of first } \\
\text { (migrated) stent } \\
\text { due to tumor in- } \\
\text { growth } 1\end{array}$ & 1 & $\begin{array}{l}\text { Peritoneal+ } \\
\text { Retroperito- } \\
\text { neal }\end{array}$ & $\begin{array}{l}\text { Nasogastric } \\
\text { suction and } \\
\text { bowel rest } \\
\text { Venting gas- } \\
\text { trostomy }\end{array}$ & Elderly; cancer \\
\hline $\begin{array}{l}\# 4,86 \text {, } \\
\text { female }\end{array}$ & $\begin{array}{l}\text { Jaundice, } \\
\text { CT abnor- } \\
\text { mality }\end{array}$ & $\begin{array}{l}\text { Cholangio- } \\
\text { carcinoma }\end{array}$ & $\begin{array}{l}\text { Common he- } \\
\text { patic duct } \\
\text { stricture }\end{array}$ & $\begin{array}{l}8.5 \mathrm{Fr}, 15 \mathrm{~cm} \text { plas- } \\
\text { tic biliary stent in } \\
\text { left hepatic duct } \\
7 \mathrm{Fr}, 15 \mathrm{~cm} \text { plastic } \\
\text { biliary stent in } \\
\text { right hepatic duct }\end{array}$ & 2 & $\begin{array}{l}\text { Retroperito- } \\
\text { neal }\end{array}$ & $\begin{array}{l}\text { Stent re- } \\
\text { moval } \\
\text { Endoscopic } \\
\text { clip (3) clo- } \\
\text { sure }\end{array}$ & $\begin{array}{l}\text { Elderly; cachexia } \\
\text { with recent } \\
\text { weight loss; can- } \\
\text { cer }\end{array}$ \\
\hline $\begin{array}{l}\# 5,71 \text {, } \\
\text { female }\end{array}$ & Jaundice & $\begin{array}{l}\text { Metastatic } \\
\text { pancreatic } \\
\text { cancer } \\
\text { with biliary } \\
\text { obstruc- } \\
\text { tion }\end{array}$ & $\begin{array}{l}\text { Distal com- } \\
\text { mon bile duct } \\
\text { stricture }+ \\
\text { right main in- } \\
\text { trahepatic } \\
\text { stricture }\end{array}$ & $\begin{array}{l}7 \mathrm{Fr}, 12 \mathrm{~cm} \text { plastic } \\
\text { biliary stent in } \\
\text { right hepatic } \\
\text { duct }^{1}\end{array}$ & 14 & Not defined & $\begin{array}{l}\text { Stent re- } \\
\text { moval } \\
\text { Endoscopic } \\
\text { clip (2) clo- } \\
\text { sure }\end{array}$ & Elderly; cancer \\
\hline $\begin{array}{l}\# 6,48, \\
\text { male }\end{array}$ & $\begin{array}{l}\text { Cholangi- } \\
\text { tis }\end{array}$ & $\begin{array}{l}\text { Necrotiz- } \\
\text { ing pan- } \\
\text { creatitis }\end{array}$ & $\begin{array}{l}\text { Common bile } \\
\text { duct stricture }\end{array}$ & $\begin{array}{l}10 \mathrm{Fr}, 12 \mathrm{~cm} \text { plas- } \\
\text { tic biliary stent in } \\
\text { common hepatic } \\
\text { duct }^{1} \\
10 \mathrm{Fr}, 10 \mathrm{~cm} \text { plastic } \\
\text { biliary stent in } \\
\text { common hepatic } \\
\text { duct }\end{array}$ & 7 & $\begin{array}{l}\text { Retroperito- } \\
\text { neal }\end{array}$ & Laparotomy & $\begin{array}{l}\text { Severe duodenal } \\
\text { edema; 5-month } \\
\text { hospitalization } \\
\text { with critical ill- } \\
\text { ness }\end{array}$ \\
\hline
\end{tabular}


$>$ Table 1 (Continuation)

\begin{tabular}{|c|c|c|c|c|c|c|c|c|}
\hline $\begin{array}{l}\text { Patient, } \\
\text { age } \\
\text { (Years), } \\
\text { Gender }\end{array}$ & $\begin{array}{l}\text { Indica- } \\
\text { tion for } \\
\text { ERCP }\end{array}$ & $\begin{array}{l}\text { Pancrea- } \\
\text { tobiliary } \\
\text { Diagnosis }\end{array}$ & $\begin{array}{l}\text { ERCP } \\
\text { Findings }\end{array}$ & $\begin{array}{l}\text { Pancreatobiliary } \\
\text { Stents Used } \\
\text { (Stent Diameter, } \\
\text { Stent Length) }\end{array}$ & $\begin{array}{l}\text { Time to } \\
\text { Perfora- } \\
\text { tion Iden- } \\
\text { tification } \\
\text { (Days } \\
\text { Post- } \\
\text { ERCP) }\end{array}$ & Location & $\begin{array}{l}\text { Treatment } \\
\text { of Perfora- } \\
\text { tion }\end{array}$ & $\begin{array}{l}\text { Potential } \\
\text { Risk Factors }\end{array}$ \\
\hline $\begin{array}{l}\# 7,61 \text {, } \\
\text { female }\end{array}$ & Jaundice & $\begin{array}{l}\text { Primary } \\
\text { sclerosing } \\
\text { cholangitis }\end{array}$ & $\begin{array}{l}\text { Multifocal in- } \\
\text { tra and extra- } \\
\text { hepatic biliary } \\
\text { strictures }\end{array}$ & $\begin{array}{l}7 \mathrm{Fr}, 18 \mathrm{~cm} \text { plastic } \\
\text { biliary stent in } \\
\text { left intrahepatic } \\
\text { duct }^{1} \\
7 \mathrm{Fr}, 15 \mathrm{~cm} \text { plastic } \\
\text { biliary stent in } \\
\text { right intrahepatic } \\
\text { duct }\end{array}$ & 70 & $\begin{array}{l}\text { Retroperito- } \\
\text { neal }\end{array}$ & $\begin{array}{l}\text { Stent re- } \\
\text { moval } \\
\text { Endoscopic } \\
\text { clip (4) clo- } \\
\text { sure }\end{array}$ & \\
\hline $\begin{array}{l}\# 8,90 \text {, } \\
\text { male }\end{array}$ & $\begin{array}{l}\text { Cholangi- } \\
\text { tis }\end{array}$ & $\begin{array}{l}\text { Cholangio- } \\
\text { carcinoma }\end{array}$ & $\begin{array}{l}\text { Left main in- } \\
\text { trahepatic } \\
\text { bile duct stric- } \\
\text { ture }\end{array}$ & $\begin{array}{l}7 \mathrm{Fr}, 13-\mathrm{cm} \text { plastic } \\
\text { biliary stent in } \\
\text { left hepatic duct }\end{array}$ & 46 & Not Defined & $\begin{array}{l}\text { Stent re- } \\
\text { moval } \\
\text { Endoscopic } \\
\text { clip (2) clo- } \\
\text { sure }\end{array}$ & Elderly; cancer \\
\hline $\begin{array}{l}\# 9,56 \text {, } \\
\text { male }\end{array}$ & $\begin{array}{l}\text { Jaundice, } \\
\text { MRCP ab- } \\
\text { normality }\end{array}$ & $\begin{array}{l}\text { Metastatic } \\
\text { cholangio- } \\
\text { carcinoma }\end{array}$ & $\begin{array}{l}\text { Bismuth IV hi- } \\
\text { lar stricture }\end{array}$ & $\begin{array}{l}10 \mathrm{Fr}, 18-\mathrm{cm} \text { plas- } \\
\text { tic biliary stent in } \\
\text { left intrahepatic } \\
\text { duct }^{1} \\
7 \mathrm{Fr}, 15 \mathrm{~cm} \text { plastic } \\
\text { biliary stent in } \\
\text { right intrahepatic } \\
\text { duct }\end{array}$ & 47 & $\begin{array}{l}\text { Retroperito- } \\
\text { neal }\end{array}$ & $\begin{array}{l}\text { Stent re- } \\
\text { moval } \\
\text { Over-the- } \\
\text { scope clip } \\
\text { (OTSC) } \\
\text { balloon to } \\
\text { sweep } \\
\text { sludge/pus }\end{array}$ & $\begin{array}{l}\text { Cancer; on che- } \\
\text { motherapy }\end{array}$ \\
\hline $\begin{array}{l}\# 10,52, \\
\text { male }\end{array}$ & Jaundice & $\begin{array}{l}\text { Cholangio- } \\
\text { carcinoma }\end{array}$ & $\begin{array}{l}\text { Bismuth IV hi- } \\
\text { lar stricture }\end{array}$ & $\begin{array}{l}10 \mathrm{Fr}, 15-\mathrm{cm} \text { plas- } \\
\text { tic transpapillary } \\
\text { biliary stent in left } \\
\text { hepatic duct } \\
7 \mathrm{Fr}, 13-\mathrm{cm} \text { plastic } \\
\text { transpapillary } \\
\text { biliary stent in } \\
\text { right anterior he- } \\
\text { patic duct }{ }^{1} \\
7 \mathrm{Fr}, 13-\mathrm{cm}^{-} \text {plastic } \\
\text { biliary stent in } \\
\text { right posterior he- } \\
\text { patic duct }\end{array}$ & 102 & Not Defined & $\begin{array}{l}\text { Stent re- } \\
\text { moval } \\
\text { Endoscopic } \\
\text { clip (3) clo- } \\
\text { sure }\end{array}$ & $\begin{array}{l}\text { Cancer; on che- } \\
\text { motherapy; } \\
\text { chronic alcoholic }\end{array}$ \\
\hline $\begin{array}{l}\# 11,88, \\
\text { male }\end{array}$ & Jaundice & $\begin{array}{l}\text { Metastatic } \\
\text { gallbladder } \\
\text { cancer } \\
\text { with biliary } \\
\text { obstruc- } \\
\text { tion }\end{array}$ & $\begin{array}{l}\text { Common he- } \\
\text { patic duct } \\
\text { stricture }\end{array}$ & $\begin{array}{l}10 \mathrm{Fr}, 9-\mathrm{cm} \text { plastic } \\
\text { transpapillary bili- } \\
\text { ary stent in right } \\
\text { intrahepatic duct } \\
\mathbf{1 0} \mathrm{Fr}, 15-\mathrm{cm} \text { plas- } \\
\text { tic transpapillary } \\
\text { biliary stent in } \\
\text { left intrahepatic } \\
\text { duct }^{1}\end{array}$ & 101 & $\begin{array}{l}\text { Retroperito- } \\
\text { neal }\end{array}$ & $\begin{array}{l}\text { Stent re- } \\
\text { moval } \\
\text { Endoscopic } \\
\text { clip (2) clo- } \\
\text { sure }\end{array}$ & $\begin{array}{l}\text { Elderly; cancer; } \\
\text { on chemother- } \\
\text { apy }\end{array}$ \\
\hline
\end{tabular}

\footnotetext{
${ }^{1}$ Biliary stent causing perforation (in bold)
} 

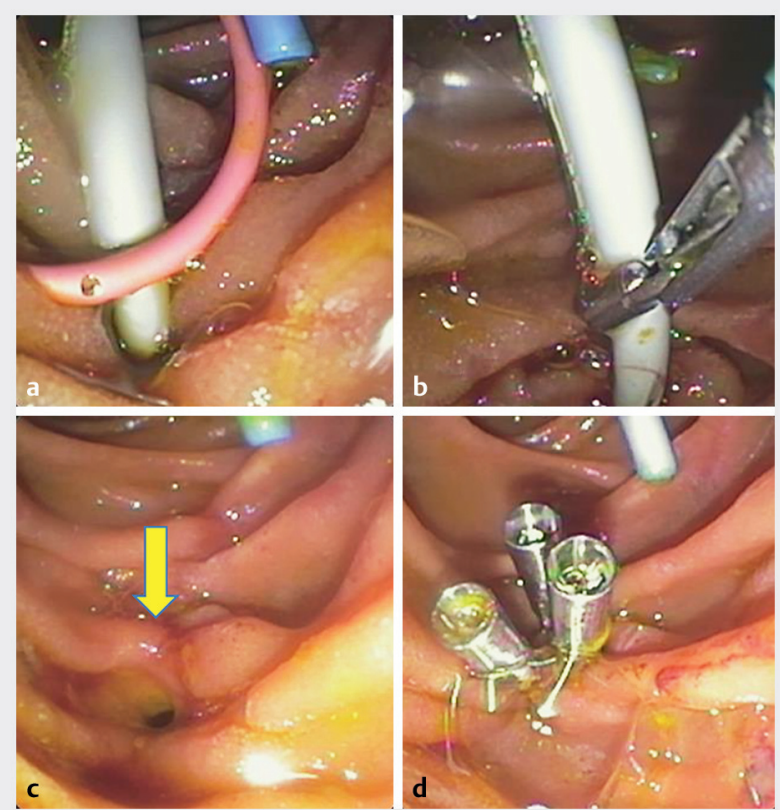

- Fig. 1 Duodenal perforation due to biliary stent migration. a Biliary stent noted to perforate the duodenal wall. b Stent removal with grasping forceps. c Identification of perforation (yellow arrow). d Closure of perforation with three endoclips.
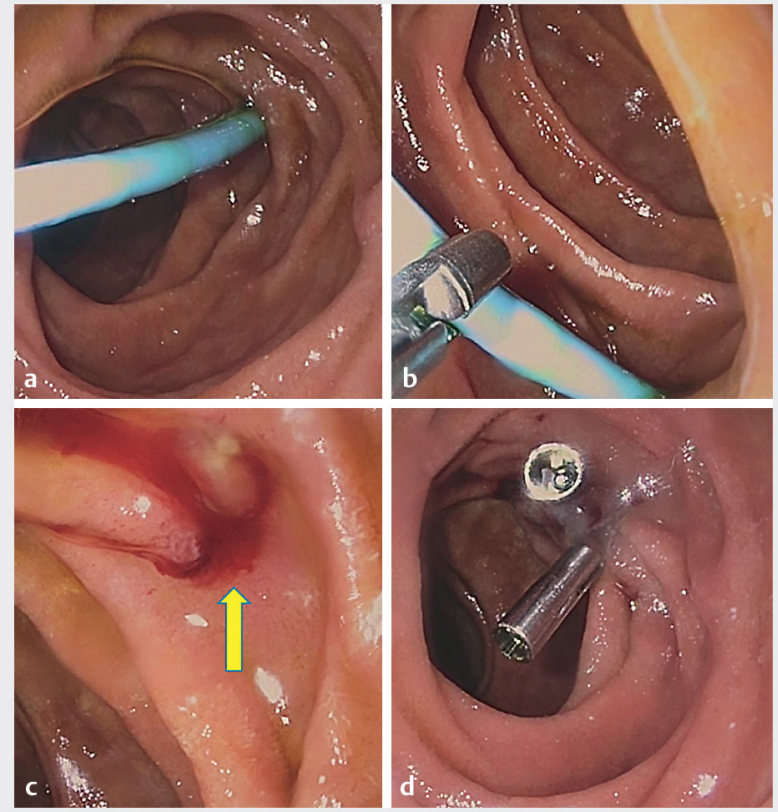

- Fig. 2 Duodenal perforation due to biliary stent migration. a Biliary stent noted to perforate the duodenal wall. $\mathbf{b}$ Stent removal with grasping forceps. c Identification of perforation (yellow arrow). d Closure of perforation with two endoclips.

stents that were implicated were plastic. The one implicated metallic biliary stent was a complex case where an original uncovered metallic self-expanding metallic biliary stent (SEMS)

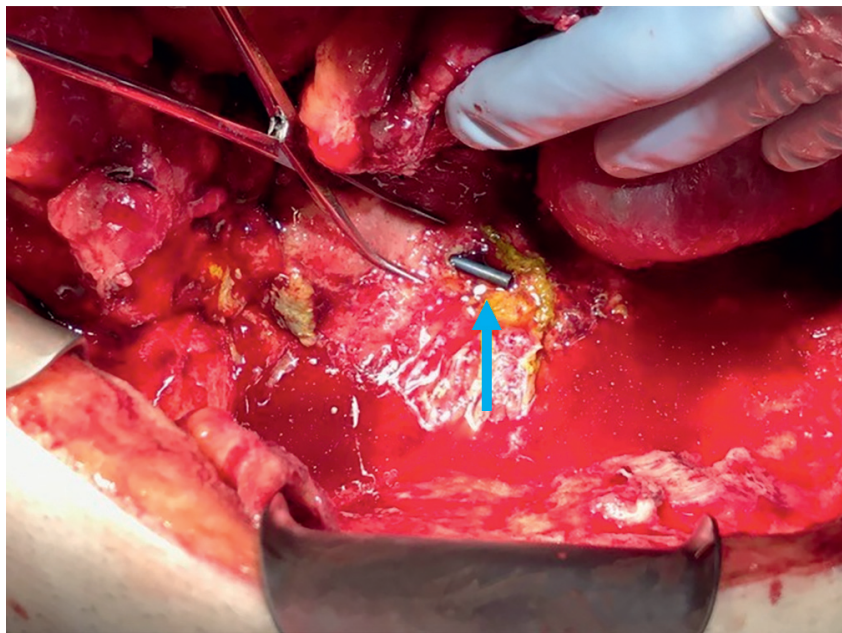

- Fig. 3 Duodenal perforation from biliary stent (blue arrow) noted intraoperatively.

was noted to have migrated distally on subsequent ERCP 11 months later. Due to the migrated metallic stent, a second metallic SEMS was placed through the interstices of the original migrated stent. This required dilation of the interstices. It is plausible that this created relatively fixed and sharp stent components that resulted in the perforation in this case.

Other potential risk factors for perforation include impaired mucosal healing and integrity. Of the 11 patients in this series, four patients with cancer were older than 85 years of age. One patient had chronic rheumatoid arthritis on multiple immunesuppressive agents (prednisone, infliximab, methotrexate), one 40 -year-old patient with cancer had received extensive chemoradiation, and another patient had metastatic pancreatic cancer with cachexia. Two patients were middle-aged men with cholangiocarcinoma on chemotherapy, one patient had severe duodenal edema and a 5-month hospitalization following severe necrotizing pancreatitis, and one patient had primary sclerosing cholangitis. These co-existing diseases or conditions in our patients may have contributed to impaired duodenal mucosal integrity. In addition, all perforating plastic stents were $\geq 9$ $\mathrm{cm}$ in length. Longer stents may facilitate leverage for a perforation with a migration event, particularly if there is an upstream stricture that may provide resistance to a migrated stent moving back upstream. Finally, the factory-made shape of the biliary stents may predispose to migration. The majority of the plastic stents causing perforation originated from the left intrahepatic ducts. At our institution, we use plastic biliary stents that are factory-groomed in the middle of the stent to conform to the shape of the right intrahepatic biliary tree. Placement of these stents into the left intrahepatic ducts may potentially increase risk for distal migration. Manually grooming the stents with heat to better conform to the shape/angulation of the duct may reduce the risk of spontaneous distal migration. In this retrospective case series, however, it is unknown how frequently this maneuver was performed.

Strategies to prevent this complication may be the use of external pigtail biliary stents to minimize mucosal trauma, adding 
- Table2 Clinical follow-up for patients with pancreatobiliary stent-induced duodenal perforation.

\begin{tabular}{|c|c|c|c|}
\hline $\begin{array}{l}\text { Patient, age } \\
\text { (Years), Gender }\end{array}$ & Pancreatobiliary Diagnosis & $\begin{array}{l}\text { Time From ERCP With } \\
\text { Perforation to Most Recent } \\
\text { Follow-up (Days) }\end{array}$ & Clinical Notes \\
\hline$\# 1,58$, female & Post-cholecystectomy biliary stricture & 1610 & $\begin{array}{l}\text { Biliary stricture since resolved with subse- } \\
\text { quent endoscopic stenting trials }\end{array}$ \\
\hline$\# 2,40$, male & $\begin{array}{l}\text { Metastatic abdominal desmoplastic small } \\
\text { round cell tumor with biliary obstruction }\end{array}$ & 386 & $\begin{array}{l}\text { Received palliative chemotherapy and } \\
\text { palliative stenting. Subsequently lost to } \\
\text { follow-up }\end{array}$ \\
\hline$\# 3,88$, female & $\begin{array}{l}\text { Pancreatic adenocarcinoma with biliary } \\
\text { obstruction }\end{array}$ & 11 & $\begin{array}{l}\text { Discharged from hospital on hospice. Lost } \\
\text { to follow-up thereafter }\end{array}$ \\
\hline$\# 4,86$, female & Cholangiocarcinoma & $19^{1}$ & Died from sepsis \\
\hline$\# 5,71$, female & $\begin{array}{l}\text { Metastatic pancreatic cancer with biliary } \\
\text { obstruction }\end{array}$ & $155^{1}$ & Died on hospice care \\
\hline$\# 6,48$, male & Necrotizing pancreatitis & 1193 & $\begin{array}{l}\text { Required three operations for severe pan- } \\
\text { creas necrosis and duodenal fistula. Now } \\
\text { rehabilitating and doing well }\end{array}$ \\
\hline$\# 7,61$, female & Primary sclerosing cholangitis & 673 & $\begin{array}{l}\text { Stricture resolved with endoscopic ther- } \\
\text { apy, maintains imaging surveillance of } \\
\text { primary sclerosing cholangitis }\end{array}$ \\
\hline$\# 8,90$, male & Cholangiocarcinoma & 613 & Indwelling biliary stent for biliary drainage \\
\hline \#9, 56, male & Cholangiocarcinoma & $31^{1}$ & Died on hospice care \\
\hline$\# 10,52$, male & Cholangiocarcinoma & 56 & $\begin{array}{l}\text { Continues to require palliative biliary } \\
\text { stenting for Bismuth IV hilar stricture }\end{array}$ \\
\hline$\# 11,88$, male & $\begin{array}{l}\text { Metastatic gallbladder cancer with biliary } \\
\text { obstruction }\end{array}$ & 73 & $\begin{array}{l}\text { On hospice and receives palliative biliary } \\
\text { stenting }\end{array}$ \\
\hline
\end{tabular}

additional internal flaps to plastic biliary stents, grooming of the plastic biliary stents (especially left intrahepatic stents) to conform to the intrahepatic ductal anatomy (as above), and increased usage of fully-covered self-expandable metal biliary stents in patients at risk for impaired duodenal mucosal integrity (e.g. history of immune suppression, prolonged illness). Due to the very low prevalence, prospective studies will likely be of limited utility to further understand this important complication.

\section{Conclusion}

In conclusion, perforation from migrated pancreatobiliary stents placed at ERCP is much more common with biliary stents than with pancreatic stents. This may be related to the angle of exit of biliary stents being more perpendicular to the opposing duodenal wall and the near exclusive use of external pigtail plastic stents in the pancreatic duct in our practice. All perforating plastic stents were $\geq 9 \mathrm{~cm}$ in length. Longer stents may provide leverage for perforation with a migration event.

\section{Acknowledgement}

"A Rare Complication of ERCP: Duodenal Perforation Due to Biliary Stent Migration." ACG 2017. October 16 $6^{\text {th }}, 2017$. Orlando, FL. Abstract and poster presentation.

\section{Competing interests}

Dr. Lehman is a consultant for Cook Medical. Dr. Sherman is a consultant for Cook Medical, Olympus America, and Boston Scientific. Dr. Gromski is a consultant for Boston Scientific. Dr. Easler is a consultant for Boston Scientific.

\section{References}

[1] Johanson JF, Schmalz MJ, Geenen JE. Incidence and risk factors for biliary and pancreatic stent migration. Gastrointest Endosc 1992; 38 : 341-346

[2] Arhan M, Odemis B, Parlak E et al. Migration of biliary plastic stents: experience of a tertiary center. Surg Endosc 2009; 23: 769-775

[3] Bharathi RS, Rao PP, Ghosh K. Intra-peritoneal duodenal perforation caused by delayed migration of endobiliary stent: a case report. Int J Surg 2008; 6: 478-480 
[4] Coppola R, Masetti R, Riccioni ME et al. Early retroduodenal perforation following endoscopic internal biliary drainage. Endoscopy 1993; 25: 255-256

[5] Issa $\mathrm{H}$, Nahawi M, Bseiso B et al. Migration of a biliary stent causing duodenal perforation and biliary peritonitis. World J Gastrointest Endosc 2013; 5: 523-526

[6] Katsinelos P, Kountouras ], Paroutoglou G et al. Migration of plastic biliary stents and endoscopic retrieval: an experience of three referral centers. Surg Laparosc Endosc Percutan Tech 2009; 19: 217-221

[7] Kriss M, Yen R, Fukami N et al. Duodenal perforation secondary to migrated biliary stent in a liver transplant patient: successful endoscopic closure with an over-the-scope clip. Gastrointest Endosc 2015; 81: 1258-1259

[8] Lo CH, Chung S, Bohmer RD. A devastating complication: duodenal perforation due to biliary stent migration. Surg Laparosc Endosc Percutan Tech 2008; 18: 608-610

[9] Melita G, Curro G, lapichino G et al. Duodenal perforation secondary to biliary stent dislocation: a case report and review of the literature. Chir Ital 2005; 57: 385-388
[10] Miller G, Yim D, Macari M et al. Retroperitoneal perforation of the duodenum from biliary stent erosion. Curr Surg 2005; 62: 512-515

[11] Nishiwaki M, Mizuno C, Yano K et al. Retroperitoneal perforation caused by migration of a pancreatic spontaneous dislodgement stent into periampullary diverticula. Intern Med 2018; 57: 351-355

[12] Paikos D, Gatopoulou A, Moschos J et al. Migrated biliary stent predisposing to fatal ERCP-related perforation of the duodenum. J Gastrointestin Liver Dis 2006; 15: 387-8

[13] Prachayakul V, Aswakul P, Kachintorn U. Duodenal perforation due to plastic stent migration successfully treated by endoscopy. Gastrointest Endosc 2012; 75: 1265-1266

[14] Roses LL, Ramirez AG, Seco AL et al. Clip closure of a duodenal perforation secondary to a biliary stent. Gastrointest Endosc 2000; 51 : 487-489

[15] Yaprak M, Mesci A, Colak T et al. Biliary stent migration with duodenal perforation. Eurasian J Med 2008; 40: 154-156 